\title{
Continued fraction inequalities for the Euler-Mascheroni constant
}

Hongmin $\mathrm{Xu}^{1}$ and $\mathrm{Xu} \mathrm{You}^{1,2^{*}}$

\section{"Correspondence:}

youxu@bipt.edu.cn

${ }^{1}$ Department of Mathematics and

Physics, Beijing Institute of

Petro-Chemical Technology, Beijing, 102617, P.R. China

${ }^{2}$ School of Mathematics and System Science, Beijing University of

Aeronautics and Astronautics,

Beijing, 100191, P.R. China

\section{Abstract}

The aim of this paper is to establish new inequalities for the Euler-Mascheroni constant by the continued fraction method.

MSC: $11 Y 60 ; 41 \mathrm{~A} 25 ; 41 \mathrm{~A} 20$

Keywords: Euler-Mascheroni constant; rate of convergence; continued fraction; Taylor's formula; harmonic sequence

\section{Introduction}

The Euler-Mascheroni constant was first introduced by Leonhard Euler (1707-1783) in 1734 as the limit of the sequence

$$
\gamma(n):=\sum_{m=1}^{n} \frac{1}{m}-\ln n
$$

There are many famous unsolved problems about the nature of this constant (see, e.g., the survey papers or books of Brent and Zimmermann [1], Dence and Dence [2], Havil [3] and Lagarias [4]). For example, it is a long-standing open problem if the Euler-Mascheroni constant is a rational number. A good part of its mystery comes from the fact that the known algorithms converging to $\gamma$ are not very fast, at least, when they are compared to similar algorithms for $\pi$ and $e$.

The sequence $(\gamma(n))_{n \in \mathbb{N}}$ converges very slowly toward $\gamma$, like $(2 n)^{-1}$. Up to now, many authors have been preoccupied with improving its rate of convergence (see, e.g., [2, 5-22] and the references therein). We list some main results as follows:

$$
\begin{aligned}
& \sum_{m=1}^{n} \frac{1}{m}-\ln \left(n+\frac{1}{2}\right)=\gamma+O\left(n^{-2}\right) \quad \text { (DeTemple [6]), } \\
& \sum_{m=1}^{n} \frac{1}{m}-\ln \frac{n^{3}+\frac{3}{2} n^{2}+\frac{227}{240}+\frac{107}{480}}{n^{2}+n+\frac{97}{240}}=\gamma+O\left(n^{-6}\right) \quad \text { (Mortici [13]), } \\
& \sum_{m=1}^{n} \frac{1}{m}-\ln \left(1+\frac{1}{2 n}+\frac{1}{24 n^{2}}-\frac{1}{48 n^{3}}+\frac{23}{5,760 n^{4}}\right) \\
& =\gamma+O\left(n^{-5}\right) \quad \text { (Chen and Mortici [5]). }
\end{aligned}
$$


Recently, Mortici and Chen [14] provided a very interesting sequence,

$$
\begin{aligned}
v(n)= & \sum_{m=1}^{n} \frac{1}{m}-\frac{1}{2} \ln \left(n^{2}+n+\frac{1}{3}\right) \\
& -\left(\frac{-\frac{1}{180}}{\left(n^{2}+n+\frac{1}{3}\right)^{2}}+\frac{\frac{8}{2,835}}{\left(n^{2}+n+\frac{1}{3}\right)^{3}}++\frac{\frac{5}{1,512}}{\left(n^{2}+n+\frac{1}{3}\right)^{4}}+\frac{\frac{592}{93,555}}{\left(n^{2}+n+\frac{1}{3}\right)^{5}}\right),
\end{aligned}
$$

and proved

$$
\lim _{n \rightarrow \infty} n^{12}(v(n)-\gamma)=-\frac{796,801}{43,783,740}
$$

Hence the rate of convergence of the sequence $(v(n))_{n \in \mathbb{N}}$ is $n^{-12}$.

Very recently, by inserting the continued fraction term in (1.1), Lu [9] introduced a class of sequences $\left(r_{k}(n)\right)_{n \in \mathbb{N}}$ (see Theorem 1$)$ and showed

$$
\begin{aligned}
& \frac{1}{72(n+1)^{3}}<\gamma-r_{2}(n)<\frac{1}{72 n^{3}}, \\
& \frac{1}{120(n+1)^{4}}<r_{3}(n)-\gamma<\frac{1}{120(n-1)^{4}} .
\end{aligned}
$$

In fact, $\mathrm{Lu}$ [9] also found $a_{4}$ without proof. In general, the continued fraction method could provide a better approximation than others, and has less numerical computations.

First, we will prove the following theorem.

Theorem 1 For the Euler-Mascheroni constant, we have the following convergent sequence:

$$
r(n)=1+\frac{1}{2}+\cdots+\frac{1}{n}-\ln n-\frac{a_{1}}{n+\frac{a_{2} n}{n+\frac{a_{3} n}{n_{+}}}},
$$

where $\left(a_{1}, a_{2}, a_{4}, a_{6}, a_{8}, a_{10}, a_{12}\right)=\left(\frac{1}{2}, \frac{1}{6}, \frac{3}{5}, \frac{79}{126}, \frac{7,230}{6,241}, \frac{4,146,631}{3,833,346}, \frac{306,232,774,533}{179,081,182,865}\right)$, and $a_{2 k+1}=-a_{2 k}$ for $1 \leq k \leq 6$.

Let

$$
R_{k}(n):=\frac{a_{1}}{n+\frac{a_{2} n}{n+\frac{a_{3} n}{n+\frac{a_{4} n}{}}}}
$$

(see the Appendix for their simple expressions) and

$$
r_{k}(n):=\sum_{m=1}^{n} \frac{1}{m}-\ln n-R_{k}(n) .
$$

For $1 \leq k \leq 13$, we have

$$
\lim _{n \rightarrow \infty} n^{k+1}\left(r_{k}(n)-\gamma\right)=C_{k}
$$


where

$$
\begin{aligned}
\left(C_{1}, \ldots, C_{13}\right)= & \left(-\frac{1}{12},-\frac{1}{72}, \frac{1}{120}, \frac{1}{200},-\frac{79}{25,200},-\frac{6,241}{3,175,200}, \frac{241}{105,840}, \frac{58,081}{22,018,248},\right. \\
& -\frac{262,445}{91,974,960},-\frac{2,755,095,121}{892,586,949,408}, \frac{20,169,451}{3,821,257,440}, \\
& \left.\frac{406,806,753,641,401}{45,071,152,103,463,200},-\frac{71,521,421,431}{5,152,068,292,800}\right) .
\end{aligned}
$$

Open problem For every $k \geq 1$, we have $a_{2 k+1}=-a_{2 k}$.

The main aim of this paper is to improve (1.3) and (1.4). We establish the following more precise inequalities.

Theorem 2 Let $r_{10}(n), r_{11}(n), C_{10}$ and $C_{11}$ be defined in Theorem 1, then

$$
\begin{aligned}
& C_{10} \frac{1}{(n+1)^{11}}<\gamma-r_{10}(n)<C_{10} \frac{1}{n^{11}}, \\
& C_{11} \frac{1}{(n+1)^{12}}<r_{11}(n)-\gamma<C_{11} \frac{1}{n^{12}} .
\end{aligned}
$$

Remark 1 In fact, Theorem 2 implies that $r_{10}(n)$ is a strictly increasing function of $n$, whereas $r_{11}(n)$ is a strictly decreasing function of $n$. Certainly, it has similar inequalities for $r_{k}(n)(1 \leq k \leq 9)$, we leave these for readers to verify. It is also should be noted that (1.4) cannot deduce the monotonicity of $r_{3}(n)$.

Remark 2 It is worth to point out that Theorem 2 provides sharp bounds for a harmonic sequence which are superior to Theorems 3 and 4 of Mortici and Chen [14].

\section{The proof of Theorem 1}

The following lemma gives a method for measuring the rate of convergence. This lemma was first used by Mortici [23, 24] for constructing asymptotic expansions or to accelerate some convergences. For proof and other details, see, e.g., [24].

Lemma 1 If the sequence $\left(x_{n}\right)_{n \in \mathbb{N}}$ is convergent to zero and there exists the limit

$$
\lim _{n \rightarrow+\infty} n^{s}\left(x_{n}-x_{n+1}\right)=l \in[-\infty,+\infty]
$$

with $s>1$, then there exists the limit

$$
\lim _{n \rightarrow+\infty} n^{s-1} x_{n}=\frac{l}{s-1} .
$$

In the sequel, we always assume $n \geq 2$.

We need to find the value $a_{1} \in \mathbb{R}$ which produces the most accurate approximation of the form

$$
r_{1}(n)=\sum_{m=1}^{n} \frac{1}{m}-\ln n-\frac{a_{1}}{n}
$$


here we note $R_{1}(n)=a_{1} / n$. To measure the accuracy of this approximation, we usually say that approximation (2.3) is better as $r_{1}(n)-\gamma$ faster converges to zero. Clearly,

$$
r_{1}(n)-r_{1}(n+1)=\ln \left(1+\frac{1}{n}\right)-\frac{1}{n+1}+\frac{a_{1}}{n+1}-\frac{a_{1}}{n} .
$$

It is well known that for $|x|<1$,

$$
\ln (1+x)=\sum_{m=1}^{\infty}(-1)^{m-1} \frac{x^{m}}{m} \quad \text { and } \quad \frac{1}{1-x}=\sum_{m=0}^{\infty} x^{m} .
$$

Developing expression (2.4) into power series expansion in $1 / n$, we obtain

$$
r_{1}(n)-r_{1}(n+1)=\left(\frac{1}{2}-a_{1}\right) \frac{1}{n^{2}}+\left(a_{1}-\frac{2}{3}\right) \frac{1}{n^{3}}+\left(\frac{3}{4}-a_{1}\right) \frac{1}{n^{4}}+O\left(\frac{1}{n^{5}}\right) .
$$

From Lemma 1, we see that the rate of convergence of the sequence $\left(r_{1}(n)-\gamma\right)_{n \in \mathbb{N}}$ is even higher than the value $s$ satisfying (2.1). By Lemma 1 , we have

(i) If $a_{1} \neq \frac{1}{2}$, then the rate of convergence of $\left(r_{1}(n)-\gamma\right)_{n \in \mathbb{N}}$ is $n^{-1}$ since

$$
\lim _{n \rightarrow \infty} n\left(r_{1}(n)-\gamma\right)=\frac{1}{2}-a_{1} \neq 0 .
$$

(ii) If $a_{1}=\frac{1}{2}$, from (2.5) we have

$$
r_{1}(n)-r_{1}(n+1)=-\frac{1}{6} \frac{1}{n^{3}}+O\left(\frac{1}{n^{4}}\right) .
$$

Hence the rate of convergence of $\left(r_{1}(n)-\gamma\right)_{n \in \mathbb{N}}$ is $n^{-2}$ since

$$
\lim _{n \rightarrow \infty} n^{2}\left(r_{1}(n)-\gamma\right)=-\frac{1}{12}
$$

We also observe that the fastest possible sequence $\left(r_{1}(n)\right)_{n \in \mathbb{N}}$ is obtained only for $a_{1}=\frac{1}{2}$. Just as Lu [9] did, we may repeat the above approach to determine $a_{1}$ to $a_{4}$ step by step. However, the computations become very difficult when $k \geq 5$. In this paper we use Mathematica software to manipulate symbolic computations.

Let

$$
r_{k}(n)=\sum_{m=1}^{n} \frac{1}{m}-\ln n-R_{k}(n),
$$

then

$$
r_{k}(n)-r_{k}(n+1)=\ln \left(1+\frac{1}{n}\right)-\frac{1}{n+1}+R_{k}(n+1)-R_{k}(n) .
$$

It is easy to get the following power series:

$$
\ln \left(1+\frac{1}{n}\right)-\frac{1}{n+1}=\sum_{m=2}^{\infty}(-1)^{m} \frac{m-1}{m} \frac{1}{n^{m}}
$$

Hence the key step is to expand $R_{k}(n+1)-R_{k}(n)$ into power series in $\frac{1}{n}$. Here we use some examples to explain our method. 
Step 1: For example, given $a_{1}$ to $a_{7}$, find $a_{8}$. Define

$$
\begin{aligned}
& R_{8}(n)=\frac{\frac{1}{2}}{n+\frac{\frac{n}{6}}{n+\frac{-\frac{n}{6}}{n+\frac{\frac{3}{5} n}{n+\frac{\frac{-3}{5} n}{n+\frac{79}{126} n}}}}} \\
& =\frac{-237+1,405 a_{8}+1,800 n+1,740 a_{8} n-630 n^{2}+3,780 a_{8} n^{2}+3,780 n^{3}}{6\left(79 a_{8}+600 a_{8} n+600 n^{2}+790 a_{8} n^{2}+1,260 a_{8} n^{3}+1,260 n^{4}\right)} .
\end{aligned}
$$

By using Mathematica software (Mathematica Program is very similar to the one given in Remark 3; however, it has a parameter $a_{8}$ ), we obtain

$$
\begin{aligned}
R_{8}(n+1)-R_{8}(n) & \\
=- & \frac{1}{2 n^{2}}+\frac{2}{3 n^{3}}-\frac{3}{4 n^{4}}+\frac{4}{5 n^{5}}-\frac{5}{6 n^{6}}+\frac{6}{7 n^{7}}-\frac{7}{8 n^{8}} \\
& +\frac{360,030-6,241 a_{8}}{396,900 n^{9}}+\frac{-346,440+24,964 a_{8}+6,241 a_{8}^{2}}{352,800 n^{10}}+O\left(\frac{1}{n^{11}}\right) .
\end{aligned}
$$

Substituting (2.8) and (2.10) into (2.7), we get

$$
\begin{aligned}
r_{8}(n)-r_{8}(n+1)= & \left(-\frac{8}{9}+\frac{360,030-6,241 a_{8}}{396,900}\right) \frac{1}{n^{9}} \\
& +\left(\frac{9}{10}+\frac{-346,440+24,964 a_{8}+6,241 a_{8}^{2}}{352,800}\right) \frac{1}{n^{10}}+O\left(\frac{1}{n^{11}}\right) .
\end{aligned}
$$

The fastest possible sequence $\left(r_{8}(n)\right)_{n \in \mathbb{N}}$ is obtained only for $a_{8}=\frac{7,230}{6,241}$. At the same time, it follows from (2.11) that

$$
r_{8}(n)-r_{8}(n+1)=\frac{58,081}{2,446,472} \frac{1}{n^{10}}+O\left(\frac{1}{n^{11}}\right)
$$

the rate of convergence of $\left(r_{8}(n)-\gamma\right)_{n \in \mathbb{N}}$ is $n^{-9}$ since

$$
\lim _{n \rightarrow \infty} n^{9}\left(r_{8}(n)-\gamma\right)=-\frac{58,081}{22,018,248}
$$

We can use the above approach to find $a_{k}(3 \leq k \leq 8)$. Unfortunately, it does not work well for $a_{9}$. Since $a_{3}=-a_{2}, a_{5}=-a_{4}$ and $a_{7}=-a_{6}$. So, we may conjecture $a_{9}=-a_{8}$. Now let us check it carefully.

Step 2: Check $a_{9}=-\frac{7,230}{6,241}$ to $a_{13}=-\frac{306,232,774,533}{179,081,182,865}$.

Let $a_{1}, \ldots, a_{9}$ and $R_{9}(n)$ be defined in Theorem 1. Applying Mathematica software, we obtain

$$
\begin{aligned}
R_{9}(n+1)-R_{9}(n)= & -\frac{1}{2 n^{2}}+\frac{2}{3 n^{3}}-\frac{3}{4 n^{4}}+\frac{4}{5 n^{5}}-\frac{5}{6 n^{6}}+\frac{6}{7 n^{7}}-\frac{7}{8 n^{8}}+\frac{8}{9} \frac{1}{n^{9}} \\
& -\frac{9}{10} \frac{1}{n^{10}}+\frac{736,265}{836,136} \frac{1}{n^{11}}+O\left(\frac{1}{n^{12}}\right)
\end{aligned}
$$


which is the desired result. Substituting (2.8) and (2.13) into (2.7), we get

$$
r_{9}(n)-r_{9}(n+1)=-\frac{262,445}{9,197,496} \frac{1}{n^{11}}+O\left(\frac{1}{n^{12}}\right)
$$

the rate of convergence of $\left(r_{9}(n)-\gamma\right)_{n \in \mathbb{N}}$ is $n^{-10}$ since

$$
\lim _{n \rightarrow \infty} n^{10}\left(r_{9}(n)-\gamma\right)=-\frac{262,445}{91,974,960}
$$

Next, we can use Step 1 to find $a_{10}$, and Step 2 to check $a_{11}$ and $a_{12}$. It should be noted that Theorem 2 will provide the other proofs for $a_{10}$ and $a_{11}$. So we omit the details here.

Finally, we check $a_{13}=-\frac{306,232,774,533}{179,081,182,865}$.

$$
\begin{aligned}
R_{13}(n+1)-R_{13}(n) \\
=-\frac{1}{2 n^{2}}+\frac{2}{3 n^{3}}-\frac{3}{4 n^{4}}+\frac{4}{5 n^{5}}-\frac{5}{6 n^{6}}+\frac{6}{7 n^{7}}-\frac{7}{8 n^{8}}+\frac{8}{9} \frac{1}{n^{9}} \\
\quad-\frac{9}{10} \frac{1}{n^{10}}+\frac{10}{11} \frac{1}{n^{11}}-\frac{11}{12} \frac{1}{n^{12}}+\frac{12}{13} \frac{1}{n^{13}}-\frac{13}{14} \frac{1}{n^{14}} \\
+\frac{1,903,648,586,623}{2,576,034,146,400} \frac{1}{n^{15}}+O\left(\frac{1}{n^{16}}\right) .
\end{aligned}
$$

Substituting (2.8) and (2.15) into (2.7), one has

$$
r_{13}(n)-r_{13}(n+1)=-\frac{500,649,950,017}{2,576,034,146,400} \frac{1}{n^{15}}+O\left(\frac{1}{n^{16}}\right)
$$

Since

$$
\lim _{n \rightarrow \infty} n^{14}\left(r_{13}(n)-\gamma\right)=-\frac{71,521,421,431}{5,152,068,292,800},
$$

thus the rate of convergence of $\left(r_{13}(n)-\gamma\right)_{n \in \mathbb{N}}$ is $n^{-14}$.

This completes the proof of Theorem 1 .

Remark 3 In fact, if the assertion $a_{13}=-\frac{306,232,774,533}{179,081,182,865}$ holds, then the other values $a_{j}$ $(1 \leq j \leq 12)$ must be true. The following Mathematica Program will generate $R_{13}(n+$ $1)-R_{13}(n)$ into power series in $\frac{1}{n}$ with order 16: Normal[Series $\left[\left(R_{13}[n+1]-R_{13}[n]\right) /\right.$. $n \rightarrow 1 / x,\{x, 0,16\}]] / . x \rightarrow 1 / n$.

Remark 4 It is a very interesting question to find $a_{k}$ for $k \geq 14$. However, it seems impossible by the above method.

\section{The proof of Theorem 2}

Before we prove Theorem 2, let us give a simple inequality by the Hermite-Hadamard inequality, which plays an important role in the proof.

Lemma 2 Let $f$ be twice derivable with $f^{\prime \prime}$ continuous. If $f^{\prime \prime}(x)>0$, then

$$
\int_{a}^{a+1} f(x) d x>f(a+1 / 2)
$$


In the sequel, the notation $P_{k}(x)$ means a polynomial of degree $k$ in $x$ with all of its nonzero coefficients positive, which may be different at each occurrence.

Let us begin to prove Theorem 2 . Note $r_{10}(\infty)=0$, it is easy to see

$$
\gamma-r_{10}(n)=\sum_{m=n}^{\infty}\left(r_{10}(m+1)-r_{10}(m)\right)=\sum_{m=n}^{\infty} f(m)
$$

where

$$
f(m)=\frac{1}{m+1}-\ln \left(1+\frac{1}{m}\right)-R_{10}(m+1)+R_{10}(m) .
$$

Let $D_{1}=\frac{2,755,095,121}{6,762,022,344}$. By using Mathematica software, we have

$$
f^{\prime}(x)+D_{1} \frac{1}{(x+1)^{13}}=-\frac{P_{19}(x)(x-1)+1,619,906,998,377 \cdots 5,270,931}{33,810,111,720 x(1+x)^{13} P_{10}^{(1)}(x) P_{10}^{(2)}(x)}<0,
$$

and

$$
f^{\prime}(x)+D_{1} \frac{1}{\left(x+\frac{1}{2}\right)^{13}}=\frac{P_{22}(x)}{4,226,263,965 x(1+x)^{2}(1+2 x)^{13} P_{10}^{(3)}(x) P_{10}^{(4)}(x)}>0 .
$$

Hence, we get the following inequalities for $x \geq 1$ :

$$
D_{1} \frac{1}{(x+1)^{13}}<-f^{\prime}(x)<D_{1} \frac{1}{\left(x+\frac{1}{2}\right)^{13}} .
$$

Applying $f(\infty)=0,(3.3)$ and Lemma 2, we get

$$
\begin{aligned}
f(m) & =-\int_{m}^{\infty} f^{\prime}(x) d x \leq D_{1} \int_{m}^{\infty}\left(x+\frac{1}{2}\right)^{-13} d x \\
& =\frac{D_{1}}{12}\left(m+\frac{1}{2}\right)^{-12} \leq \frac{D_{1}}{12} \int_{m}^{m+1} x^{-12} d x .
\end{aligned}
$$

From (3.1) and (3.4) we obtain

$$
\begin{aligned}
\gamma-r_{10}(n) & \leq \sum_{m=n}^{\infty} \frac{D_{1}}{12} \int_{m}^{m+1} x^{-12} d x \\
& =\frac{D_{1}}{12} \int_{n}^{\infty} x^{-12} d x=\frac{D_{1}}{132} \frac{1}{n^{11}} .
\end{aligned}
$$

Similarly, we also have

$$
\begin{aligned}
f(m) & =-\int_{m}^{\infty} f^{\prime}(x) d x \geq D_{1} \int_{m}^{\infty}(x+1)^{-13} d x \\
& =\frac{D_{1}}{12}(m+1)^{-12} \geq \frac{D_{1}}{12} \int_{m+1}^{m+2} x^{-12} d x
\end{aligned}
$$


and

$$
\begin{aligned}
\gamma-r_{10}(n) & \geq \sum_{m=n}^{\infty} \frac{D_{1}}{12} \int_{m+1}^{m+2} x^{-12} d x \\
& =\frac{D_{1}}{12} \int_{n+1}^{\infty} x^{-12} d x=\frac{D_{1}}{132} \frac{1}{(n+1)^{11}}
\end{aligned}
$$

Combining (3.5) and (3.6) completes the proof of (1.6).

Note $r_{11}(\infty)=0$, it is easy to deduce

$$
r_{11}(n)-\gamma=\sum_{m=n}^{\infty}\left(r_{11}(m)-r_{11}(m+1)\right)=\sum_{m=n}^{\infty} g(m)
$$

where

$$
g(m)=\ln \left(1+\frac{1}{m}\right)-\frac{1}{m+1}-R_{11}(m)+R_{11}(m+1) .
$$

We write $D_{2}=\frac{20,169,451}{24,495,240}$. By using Mathematica software, we have

$$
-g^{\prime}(x)-D_{2} \frac{1}{(x+1)^{14}}=\frac{P_{18}(x)}{24,495,240 x^{3}(1+x)^{14} P_{8}^{(1)}(x) P_{8}^{(2)}(x)}>0
$$

and

$$
\begin{aligned}
-g^{\prime}(x)-D_{2} \frac{1}{\left(x+\frac{1}{2}\right)^{14}} & =-\frac{P_{19}(x)(x-1)+4,622,005,677,839,353,997,724,676,307,741}{6,123,810 x^{3}(1+x)^{3}(1+2 x)^{14} P_{8}^{(3)}(x) P_{8}^{(4)}(x)} \\
& <0 .
\end{aligned}
$$

Hence, for $x \geq 1$,

$$
D_{2} \frac{1}{(x+1)^{14}}<-g^{\prime}(x)<D_{2} \frac{1}{\left(x+\frac{1}{2}\right)^{14}} .
$$

Applying $g(\infty)=0,(3.8)$ and (3.1), we get

$$
\begin{aligned}
g(m) & =-\int_{m}^{\infty} g^{\prime}(x) d x \leq D_{2} \int_{m}^{\infty}\left(x+\frac{1}{2}\right)^{-14} d x \\
& =\frac{D_{2}}{13}\left(m+\frac{1}{2}\right)^{-13} \leq \frac{D_{2}}{13} \int_{m}^{m+1} x^{-13} d x .
\end{aligned}
$$

It follows from (3.7) and (3.9) that

$$
\begin{aligned}
r_{11}(n)-\gamma & \leq \sum_{m=n}^{\infty} \frac{D_{2}}{13} \int_{m}^{m+1} x^{-13} d x \\
& =\frac{D_{2}}{13} \int_{n}^{\infty} x^{-13} d x=\frac{D_{2}}{156} \frac{1}{n^{12}} .
\end{aligned}
$$


Finally,

$$
\begin{aligned}
g(m) & =-\int_{m}^{\infty} g^{\prime}(x) d x \geq D_{2} \int_{m}^{\infty}(x+1)^{-14} d x \\
& =\frac{D_{2}}{13}(m+1)^{-13} \geq \frac{D_{2}}{13} \int_{m+1}^{m+2} x^{-13} d x
\end{aligned}
$$

and

$$
\begin{aligned}
r_{11}(n)-\gamma & \geq \sum_{m=n}^{\infty} \frac{D_{2}}{13} \int_{m+1}^{m+2} x^{-13} d x \\
& =\frac{D_{2}}{13} \int_{n+1}^{\infty} x^{-13} d x=\frac{D_{2}}{156} \frac{1}{(n+1)^{12}} .
\end{aligned}
$$

Combining (3.10) and (3.11) completes the proof of (1.7).

Remark 5 As an example, we give Mathematica Program for the proof of the left-hand side of (3.3):

(i) Together $\left[D[f[x],\{x, 1\}]+D_{1}(x+1)^{13}\right]$;

(ii) Take out the numerator $P[x]$ of the above rational function, then manipulate the program: Apart $[P[x] /(x-1)]$.

\section{Appendix}

For the reader's convenience, we rewrite $R_{k}(n)(k \leq 13)$ with minimal denominators as follows.

$$
\begin{aligned}
& R_{1}(n)=\frac{1}{2 n} \\
& R_{3}(n)=\frac{1}{2 n}-\frac{1}{12} \frac{1}{n^{2}} \\
& R_{5}(n)=\frac{1}{2 n}-\frac{5}{6\left(1+10 n^{2}\right)}, \\
& R_{7}(n)=\frac{1}{2 n}-\frac{79}{1,200} \frac{1}{n^{2}}-\frac{147}{400\left(10+21 n^{2}\right)}, \\
& R_{9}(n)=\frac{1}{2 n}-\frac{7\left(871+790 n^{2}\right)}{20\left(241+3,990 n^{2}+3,318 n^{4}\right)}, \\
& R_{11}(n)=\frac{1}{2 n}-\frac{52,489}{894,348} \frac{1}{n^{2}}-\frac{1,237,227,621+584,280,400 n^{2}}{4,471,740\left(3,549+13,020 n^{2}+5,302 n^{4}\right)}, \\
& R_{13}(n)=\frac{1}{2 n}-\frac{39,577,260,671+66,288,226,620 n^{2}+15,762,446,700 n^{4}}{1,260\left(20,169,451+434,410,620 n^{2}+646,328,298 n^{4}+150,118,540 n^{6}\right)} \\
& R_{2}(n)=\frac{3}{6 n+1}, \\
& R_{4}(n)=\frac{13+30 n}{6\left(1+6 n+10 n^{2}\right)}, \\
& 5\left(281+348 n+756 n^{2}\right)
\end{aligned}
$$




$$
\begin{aligned}
R_{8}(n)= & \frac{964,337+2,646,000 n+2,599,730 n^{2}+2,621,220 n^{3}}{20\left(19,039+144,600 n+315,210 n^{2}+303,660 n^{3}+262,122 n^{4}\right)} \\
R_{10}(n)= & \left(7 \left(108,237,701+208,886,046 n+523,341,290 n^{2}\right.\right. \\
& \left.\left.+210,464,400 n^{3}+230,000,760 n^{4}\right)\right) \\
& /\left(2 0 \left(12,649,849+107,768,934 n+209,431,110 n^{2}\right.\right. \\
& \left.\left.+395,365,320 n^{3}+174,158,502 n^{4}+161,000,532 n^{5}\right)\right) \\
R_{12}(n)= & (3,604,759,235,968,501+11,032,319,618,513,046 n \\
& +17,366,281,558,290,420 n^{2}+19,958,033,982,902,400 n^{3} \\
& \left.+7,661,417,445,218,460 n^{4}+4,964,130,389,017,800 n^{5}\right) \\
& /(1,260(1,058,674,313,539+9,019,254,081,474 n \\
& +22,801,779,033,180 n^{2}+33,088,387,754,520 n^{3}+33,925,126,033,722 n^{4} \\
& \left.\left.+13,474,242,079,452 n^{5}+7,879,572,046,060 n^{6}\right)\right) .
\end{aligned}
$$

\section{Competing interests}

The authors declare that they have no competing interests.

\section{Authors' contributions}

All authors read and approved the final manuscript.

\section{Acknowledgements}

The authors would like to thank the referees and Prof Xiaodong Cao for their careful reading of the manuscript and insightful comments. Research of this paper was supported by the National Natural Science Foundation of China (Grant No.11171344) and the Natural Science Foundation of Beijing (Grant No.1112010).

\section{Received: 25 June 2014 Accepted: 21 August 2014 Published: 03 Sep 2014}

\section{References}

1. Brent, RP, Zimmermann, P: Modern Computer Arithmetic. Cambridge Monographs on Applied and Computational Mathematics, vol. 18, p. xvi+221. Cambridge University Press, Cambridge (2011)

2. Dence, TP, Dence, JB: A survey of Euler's constant. Math. Mag. 82, 255-265 (2009)

3. Havil, J: Gamma: Exploring Euler's Constant. Princeton University Press, Princeton (2003)

4. Lagarias, JC: Euler's constant: Euler's work and modern developments. Bull. Am. Math. Soc. (N.S.) 50(4), 527-628 (2013)

5. Chen, CP, Mortici, C: New sequence converging towards the Euler-Mascheroni constant. Comput. Math. Appl. 64, 391-398 (2012)

6. DeTemple, DW: A quicker convergence to Euler's constant. Am. Math. Mon. 100(5), 468-470 (1993)

7. Gavrea, I, Ivan, M: Optimal rate of convergence for sequences of a prescribed form. J. Math. Anal. Appl. 402(1), 35-43 (2013)

8. Gourdon, X, Sebah, P: Collection of formulae for the Euler constant. http://numbers.computation.free.fr/Constants/Gamma/gammaFormulas.pdf, or see, http://numbers.computation.free.fr/Constants/constants.html

9. Lu, D: A new quicker sequence convergent to Euler's constant. J. Number Theory 136, 320-329 (2014)

10. Lu, D: Some quicker classes of sequences convergent to Euler's constant. Appl. Math. Comput. 232, 172-177 (2014)

11. Lu, D: Some new convergent sequences and inequalities of Euler's constant. J. Math. Anal. Appl. 419(1), 541-552 (2014)

12. Lu, D: Some new improved classes of convergence towards Euler's constant. Appl. Math. Comput. 243, 24-32 (2014)

13. Mortici, C: On new sequences converging towards the Euler-Mascheroni constant. Comput. Math. Appl. 59(8), 2610-2614 (2010)

14. Mortici, C, Chen, CP: On the harmonic number expansion by Ramanujan. J. Inequal. Appl. 2013, 222 (2013)

15. Mortici, C: Optimizing the rate of convergence in some new classes of sequences convergent to Euler's constant. Anal. Appl. 8(1), 99-107 (2010)

16. Mortici, C: A quicker convergence toward the gamma constant with the logarithm term involving the constant $e$. Carpath. J. Math. 26(1), 86-91 (2010)

17. Mortici, C: A continued fraction approximation of the gamma function. J. Math. Anal. Appl. 402(2), $405-410$ (2013)

18. Mortici, C, Berinde, V: New sharp estimates of the generalized Euler-Mascheroni constant. Math. Inequal. Appl. 16(1), 279-288 (2013)

19. Mortici, C: Fast convergences toward Euler-Mascheroni constant. Comput. Appl. Math. 29(3), $479-491$ (2010) 
20. Mortici, C: On some Euler-Mascheroni type sequences. Comput. Math. Appl. 60(7), 2009-2014 (2010)

21. Mortici, C: On new sequences converging towards the Euler-Mascheroni constant. Comput. Math. Appl. 59(8), 2610-2614 (2010)

22. Mortici, C: Improved convergence towards generalized Euler-Mascheroni constant. Appl. Math. Comput. 215(9), 3443-3448 (2010)

23. Mortici, C: Product approximations via asymptotic integration. Am. Math. Mon. 117(5), 434-441 (2010)

24. Mortici, C: New approximations of the gamma function in terms of the digamma function. Appl. Math. Lett. 23, 97-100 (2010)

10.1186/1029-242X-2014-343

Cite this article as: $\mathrm{Xu}$ and You: Continued fraction inequalities for the Euler-Mascheroni constant. Journal of Inequalities and Applications 2014, 2014:343

Submit your manuscript to a SpringerOpen ${ }^{\circ}$ journal and benefit from:

- Convenient online submission

Rigorous peer review

- Immediate publication on acceptance

- Open access: articles freely available online

- High visibility within the field

- Retaining the copyright to your article

Submit your next manuscript at springeropen.com 